\title{
Surgery on a pair of transversal manifolds
}

\author{
A. Bak · Y. V. Muranov
}

Received: 18 September 2011 / Accepted: 21 April 2012 / Published online: 30 May 2012

(C) Tbilisi Centre for Mathematical Sciences 2012

\begin{abstract}
In the paper we construct the algebraic surgery theory of a topological space consisting of two manifolds which intersect transversally. Such a space is a basic example of stratified space. For such spaces, we define the notion of a normal map, an $s$-triangulation, and surgery obstruction groups, and show that these notions can be realized on the level of spectra. We construct commutative braids of exact sequences which relate the new notions to their classical counterparts. We examine the example of a transversal intersection of two real projective spaces and obtain explicit results regarding structure sets in this situation.
\end{abstract}

Keywords Surgery obstruction groups - Splitting obstruction groups · Stratified manifolds $\cdot$ Surgery exact sequence

Mathematics Subject Classification $\quad 57 \mathrm{R} 67 \cdot 19 \mathrm{~J} 25 \cdot 57$ N99

\section{Introduction}

The surgery theory of Milnor-Novikov-Browder-Sullivan-Wall is an effective tool for classifying manifolds in anyone of the categories TOP, PL, or Diff, which are homotopy equivalent to a given manifold or Poincaré complex [12]. The generalization

Communicated by Guoping Tang.

A. Bak (ه)

Department of Mathematics, University of Bielefeld, 33501 Bielefeld, Germany

e-mail: bak@mathematik.uni-bielefeld.de

Y. V. Muranov

Institute of Mathematics, Jagiellonian University, 30-348 Kraków, Poland

e-mail: ymuranov@mail.ru 
of this theory, which we develop, can be applied to classifying stratified spaces (see, for example $[4,5,13]$.

In the current paper we construct the algebraic surgery theory of a stratified space $\mathcal{X}=\left(X_{1}, X_{2} ; X_{1} \cap X_{2}=Y\right)$ of two manifolds $X_{1}, X_{2}$ with transversal intersection $Y$. Although we consider only the case $\operatorname{dim} X_{1}=\operatorname{dim} X_{2}$, our result can be extended to the situation that $\operatorname{dim} X_{1}$ and $\operatorname{dim} X_{2}$ are arbitrary. Under the assumption that $\operatorname{dim} X_{1}=\operatorname{dim} X_{2}$, we define the notions of normal map, $s$-triangulation, $t$-triangulation, and surgery obstruction groups, and show that the notions can be realized on the level of spectra. We construct commutative braids of exact sequences which relate the new notions with their classical counterparts for manifold pairs $Y \subset X_{1}$ and $Y \subset X_{2}$. We consider the example of a transversal intersection of two real projective spaces and obtain explicit results regarding the structure sets in this situation. The main results are given in Theorems 3.2, 3.3, 3.4, 3.6, and 3.7.

The rest of the paper is organized as follows. In Sect. 2, we provide preliminary material regarding the algebraic surgery and splitting theory (see [2-4,6,10-12]).

In Sect. 3, we construct the algebraic surgery theory of a stratified space $\mathcal{X}$ of two manifolds with a transversal intersection $Y$.

In Sect. 4, we show that one can compute structure sets by treating the example of a transversal intersection of two real projective spaces.

\section{Algebraic L-theory for a manifold pair}

In this section we recall the algebraic surgery theory of manifold pairs (see $[2,3,6,10-$ 12]). Throughout the section, $X$ denotes a compact or closed $n$-dimensional manifold, $n \geq 5$, and for a manifold pair $Y^{n-q} \subset X$, we shall suppose that $Y$ is a locally flat topological submanifold equipped with the structure of a normal topological bundle (see [11, pages 562-563]).

Let $X^{n}, M^{n}$ be closed $n$-dimensional manifolds. A simple homotopy equivalence $f: M \rightarrow X$ is called an $s$-triangulation of the manifold $X$. Two $s$-triangulations

$$
f_{i}: M_{i} \rightarrow X, i=1,2
$$

are equivalent if there exists a homeomorphism $g: M_{1} \rightarrow M_{2}$ such that $f_{2} g$ is homotopic to $f_{1}$. The set of equivalence classes of $s$-triangulations is denoted by $\mathcal{S}^{T O P}(X)$.

Let $X^{n}, M^{n}$ be closed $n$-dimensional manifolds. Let $v_{M}$ be a stable normal bundle over $M$ and $\xi_{X}$ a stable bundle over $X$. A $t$-triangulation of $X$ is a pair $(f, b)$ of maps

$$
f: M \rightarrow X, b: v_{M} \rightarrow \xi_{X}
$$

where $f$ is any degree one continuous map and $b$ is a map of stable bundles over $f$. (A $t$-triangulation is often called a normal map in the literature). The set of concordance, i.e. normal cobordism, classes of normal maps is denoted by $\mathcal{T}^{T O P}(X)$. $\left(\mathcal{T}^{T O P}(X)\right.$ is often called in the literature the set of normal invariants of $X$.)

Let $\pi=\pi_{1}(X)$ denote the fundamental group of $X$ equipped with an orientation character $w: \pi \rightarrow\{ \pm 1\}$. The structure sets $\mathcal{S}^{T O P}(X)$ and $\mathcal{T}^{T O P}(X)$ fit into the 
Wall-Novikov-Sullivan surgery exact sequence (see [9] and [10, §10])

$$
\cdots \rightarrow L_{n+1}(\pi) \rightarrow \mathcal{S}^{T O P}(X) \rightarrow[X, G / T O P] \rightarrow L_{n}(\pi)
$$

where $[X, G / T O P]$ is isomorphic to the set of normal invariants $\mathcal{T}^{T O P}(X)$ and $L_{n}(\pi)=L_{n}(\pi, w), i=0,1,2,3 \bmod 4$ are the surgery obstruction groups. Since the members of the structure set $\mathcal{S}^{T O P}$ are defined by simple homotopy equivalences, the surgery groups $L_{n}$ above are often decorated by a superscript $s$, yielding $L_{n}^{s}$. The surgery exact sequence (2.2) is isomorphic to the homotopy long exact sequence in dimensions $\geq n$ of the cofibration of spectra (see [10, p. 276] and [11])

$$
X_{+} \wedge \mathbf{L}_{\bullet} \rightarrow \mathbb{L}(\pi) \rightarrow \mathbb{S}(X)
$$

where $\mathbf{L}_{\bullet}$ is the 1-connected cover of the spectrum $\mathbb{L}(1)$ with $\mathbf{L}_{\bullet 0} \simeq G / T O P$ and the surgery obstruction groups $L_{i}(\pi)=L_{i}(\pi, w), i=0,1,2,3 \bmod 4$ are realized by the $\Omega$-spectrum

$$
\mathbb{L}(\pi, w)=\mathbb{L}(\pi)=\left\{\mathbb{L}_{-k}(\pi): k \in \mathbb{Z}\right\}, L_{n}(\pi)=\pi_{n}(\mathbb{L}(\pi)) .
$$

In this case, we have

$$
H_{n}\left(X ; \mathbf{L}_{\bullet}\right) \cong[X, G / T O P] \cong \mathcal{T}^{T O P}(X)
$$

and

$$
\mathbb{S}_{m}(X)=\pi_{m}(\mathbb{S}(X)), \mathcal{S}_{n+1}(X) \cong \mathcal{S}^{T O P}(X) .
$$

Note that the cofibration (2.3) is defined functorially for any topological space $X$ with $\pi_{1}(X)=\pi$.

Let $(X, \partial X),(M, \partial M)$ denote compact $n$-dimensional manifolds with boundary. Let $v_{M}$ be a stable normal bundle over $M$ and $\xi_{X}$ a stable bundle over $X$. A $t$-triangulation of a manifold with boundary $(X, \partial X)$ is a pair $((f, b),(\partial f, \partial b))$ of pairs $(f, b)$ and $(\partial f, \partial b)$ where the pair

$$
f: M \rightarrow X, b: v_{M} \rightarrow \xi_{X}
$$

satisfies $f$ is any continuous map and $b$ is a map of stable bundles over $f$ and the pair

$$
\partial f=\left.f\right|_{\partial M}: \partial M \rightarrow \partial X, \partial b=\left.b\right|_{\left(\left.v_{M}\right|_{\partial M}\right)}:\left.\left.v_{M}\right|_{\partial M} \rightarrow \xi_{X}\right|_{\partial X}
$$

is a $t$-triangulation of $\partial X$ (note that $\partial X$ and $\partial M$ are necessary closed manifolds).

Two $t$-triangulations

$$
\left(\left(f_{i}, b_{i}\right),\left(\partial f_{i}, \partial b_{i}\right)\right):\left(M_{i}, \partial M_{i}\right) \rightarrow(X, \partial X), i=0,1
$$


are concordant (see $[6,11])$ if there exists a $t$-triangulation

$$
((F, B),(\partial F, \partial B)):(W, \partial W) \rightarrow(X \times I, \partial(X \times I))
$$

of the manifold with boundary $(X \times I, \partial(X \times I))$ such that

$$
\begin{gathered}
\partial W=M_{0} \cup_{\partial M_{0}} V \cup_{\partial M_{1}} M_{1}, \partial V=\partial M_{0} \cup \partial M_{1}, \\
\left.F\right|_{M_{i}}=f_{i},\left.B\right|_{\nu_{M_{i}}}=b_{i}, \quad i=0,1 .
\end{gathered}
$$

In this situation, we shall say that we have a normal map of 4-ads and write

$$
\begin{aligned}
&((F, B) ;(g, c),\left.\left(f_{0}, b_{0}\right),\left(f_{1}, b_{1}\right)\right):\left(W ; V, M_{0}, M_{1}\right) \\
& \rightarrow(X \times I ; \partial X \times I, X \times\{0\}, X \times\{1\}),
\end{aligned}
$$

where

$$
(g, c): V \rightarrow \partial X \times I
$$

is the restriction of the normal map $(F, B)$. The set of concordance classes is denoted by $\mathcal{T}^{T O P}(X, \partial X)$.

A $t_{\partial}$-triangulation of a manifold with boundary $(X, \partial X)$ is a $t$-triangulation

$$
((f, b),(\partial f, \partial b)):(M, \partial M) \rightarrow(X, \partial X)
$$

of a manifold with boundary such that $\left.f\right|_{\partial M}=\partial f$ is a homeomorphism $\partial M \rightarrow \partial X$. Two $t_{\partial}$-triangulations

$$
\left(\left(f_{i}, b_{i}\right),\left(\partial f_{i}, \partial b_{i}\right)\right):\left(M_{i}, \partial M_{i}\right) \rightarrow(X, \partial X), i=0,1
$$

are concordant (see $[6,11]$ ) if there exists a $t$-triangulation of the $4-a d(2.4)$ such that

$$
V=\partial M_{0} \times I \text { and } g=\partial f_{0} \times I: V \rightarrow \partial X \times I .
$$

Since $\partial f_{0}$ is a homeomorphism so is $g$. The set of concordance classes is denoted by $\mathcal{T}_{\partial}^{T O P}(X, \partial X)$.

An $s$-triangulation of a manifold with boundary $(X, \partial X)$ is a simple homotopy equivalence of manifolds with boundary (see $[6,10,11])$

$$
(f, \partial f):(M, \partial M) \rightarrow(X, \partial X) .
$$


This means that $\partial f=\left.f\right|_{\partial M}$ and the maps $f$ and $\partial f$ are simple homotopy equivalences. Two $s$-triangulations

$$
\left(f_{i}, \partial f_{i}\right):\left(M_{i}, \partial M_{i}\right) \rightarrow(X, \partial X), i=0,1
$$

are concordant, i.e. cobordant, if there exists a simple homotopy equivalence

$$
\left(F ; g, f_{0}, f_{1}\right):\left(W ; V, M_{0}, M_{1}\right) \rightarrow(X \times I ; \partial X \times I, X \times\{0\}, X \times\{1\})
$$

of 4-ads with

$$
\partial W=M_{0} \cup_{\partial M_{0}} V \cup_{\partial M_{1}} M_{1}, \quad \partial V=\partial M_{0} \cup \partial M_{1} .
$$

This means that $g=\left.F\right|_{V}, f_{i}=\left.F\right|_{M_{i}}$, and $F, g, f_{i}$ are simple homotopy equivalences. The set of concordance classes is denoted by $\mathcal{S}^{T O P}(X, \partial X)$. Note that if $\partial X=\varnothing$ then $\mathcal{S}^{T O P}(X, \partial X)=\mathcal{S}^{T O P}(X)$ by the $s$-cobordism theorem [8].

An $s_{\partial}$-triangulation of a manifold with boundary $(X, \partial X)$ is an $s$-triangulation

$$
(f, \partial f):(M, \partial M) \rightarrow(X, \partial X)
$$

of a manifold with boundary such that $\left.f\right|_{\partial M}=\partial f$ is a homeomorphism $\partial M \rightarrow \partial X$. Two $s_{\partial}$-triangulations

$$
\left(f_{i}, \partial f_{i}\right):\left(M_{i}, \partial M_{i}\right) \rightarrow(X, \partial X), i=0,1
$$

are concordant (see $[6,11]$ ) if there exists an $s$-triangulation of the $4-$ ad (2.4) such that

$$
V=\partial M_{0} \times I \text { and } g=\partial f_{0} \times I: V \rightarrow \partial X \times I .
$$

Since $\partial f_{0}$ is a homeomorphism, so is $g$. The set of concordance classes is denoted $\mathcal{S}_{\partial}^{T O P}(X, \partial X)$.

The natural inclusion $\partial X \rightarrow X$ induces a map of cofibrations in (2.3) such that we obtain a homotopy commutative diagram of spectra

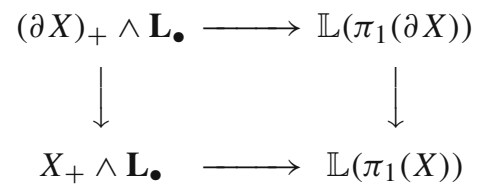

which we can extend (see [9, Lemma 1]) to the following homotopy commutative diagram of spectra 


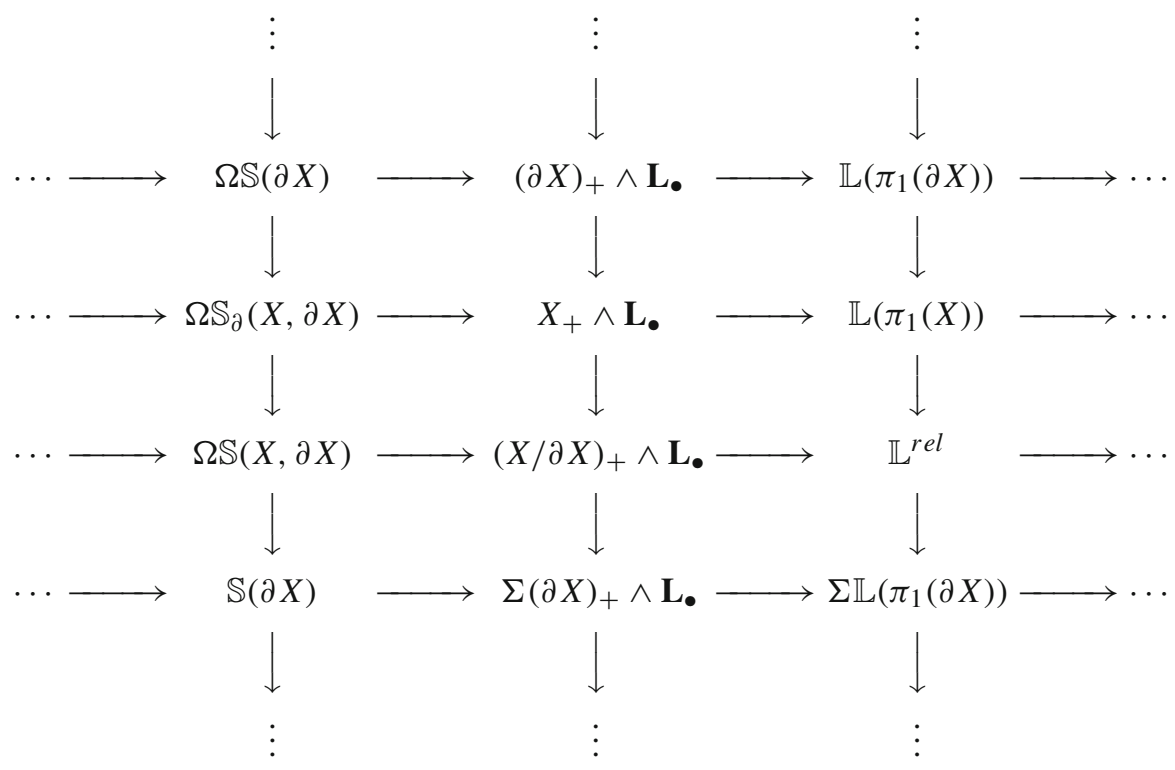

where $\mathbb{L}^{r e l}=\mathbb{L}\left(\pi_{1}(\partial X) \rightarrow \pi_{1}(X)\right)$ is the spectrum in [10] for relative $L$-groups and $\Sigma$ is the suspension functor.

The homotopy long exact sequences of any three consecutive rows of (2.5) are the usual algebraic surgery exact sequences for the manifold with boundary $(X, \partial X)$. We shall use the following notation:

$$
\begin{aligned}
& \mathcal{T}_{\partial}^{T O P}(X, \partial X)=\pi_{n}\left(X_{+} \wedge \mathbf{L}_{\bullet}\right), \mathcal{T}^{T O P}(X, \partial X)=\pi_{n}\left((X / \partial X)_{+} \wedge \mathbf{L}_{\bullet}\right), \\
& \mathcal{S}_{\partial}^{T O P}(X, \partial X)=\pi_{n+1}\left(\mathbb{S}_{\partial}(X, \partial X)\right), \mathcal{S}^{T O P}(X, \partial X)=\pi_{n+1}(\mathbb{S}(X, \partial X)) .
\end{aligned}
$$

Let $X^{n}$ be a closed $n$-dimensional manifold and let $Y^{n-q}$ be a closed submanifold of codimension $q$. A pair $(X, Y)=\left(X^{n}, Y^{n-q}, \xi\right)$ is called a closed manifold pair (see [2,3,6] and [11, page 570]), if $Y$ is a locally flat submanifold of $X$ and is equipped with a topological normal block bundle $\xi$.

A $t$-triangulation of a closed manifold pair $(X, Y)$ [10, page 570]

$$
(f, g):(M, N) \rightarrow(X, Y)
$$

is a $t$-triangulation (2.1) $f: M \rightarrow X$ such that $N=f^{-1}(Y)$ is a transversal preimage of $Y, g=\left.f\right|_{N}$, and $(M, N)=(M, N, v)$ is a closed manifold pair with the block bundle $v$ induced from $\xi$. Let $Q=X \backslash Y$ be the compliment of the submanifold $Y$ in $X$ with the boundary $\partial(X \backslash Y)=\partial Q=S(\xi)$ and let $P=M \backslash N$ be the compliment of the submanifold $N$ in $M$ with the boundary $\partial(M \backslash N)=\partial P=S(\nu)$ [10]. It is easy to show that the $t$-triangulation (2.6) has the following properties: 
(i) the restriction

$$
g=\left.f\right|_{N}: N \rightarrow Y
$$

is a $t$-triangulation of the manifold $Y$;

(ii) the restriction

$$
(h, \partial h)=\left(\left.f\right|_{P}, \partial\left(f_{P}\right)\right):(P, \partial P) \rightarrow(Q, \partial Q)
$$

is a $t$-triangulation of the manifold with boundary $(Q, \partial Q)$;

(iii) the restriction $\partial h$ coincides with the map

$$
\partial g^{!}: S(v) \rightarrow S(\xi)
$$

where the map

$$
\left(g^{!}, \partial g^{!}\right):(E(v), S(v)) \rightarrow(E(\xi), S(\xi))
$$

is induced by transfer and $f=g^{!} \cup_{\partial h} h$.

We have a natural isomorphism $[11, \S 7.2]$

$$
\mathcal{T}^{T O P}(X, Y) \stackrel{\cong}{\rightarrow} \mathcal{T}^{T O P}(X)
$$

and a natural map

$$
\mathcal{T}^{\text {TOP }}(X) \rightarrow \mathcal{T}^{\text {TOP }}(Y)
$$

which is given by restricting the normal map. This map is realized on the spectra level (see $[1,2,6,11])$ by the map of spectra

$$
X_{+} \wedge \mathbf{L}_{\bullet} \rightarrow \Sigma^{q}\left(Y_{+} \wedge \mathbf{L}_{\bullet}\right)
$$

which fits into the cofibration

$$
(X \backslash Y)_{+} \wedge \mathbf{L}_{\bullet} \rightarrow X_{+} \wedge \mathbf{L}_{\bullet} \rightarrow \Sigma^{q}\left(Y_{+} \wedge \mathbf{L}_{\bullet}\right)
$$

An $s$-triangulation of the closed manifold pair $(X, Y)[11$, page 571] is a $t$-triangulation of the pair $(X, Y)$ such that

$$
f: M \rightarrow X, g=\left.f\right|_{N}: N \rightarrow Y, \text { and } h=\left.f\right|_{P}:(P, S(\nu)) \rightarrow(Q, S(\xi))
$$

are simple homotopy equivalences. Two $s$-triangulations of closed manifold pairs

$$
\left(f_{i} ; g_{i}, h_{i}\right):\left(M_{i} ; N_{i}, M_{i} \backslash N_{i}\right) \rightarrow(X ; Y, X \backslash Y), i=0,1
$$


are concordant, if there exists a triple of simple homotopy equivalences

$$
(F ; G, H):(W ; V, W \backslash V) \rightarrow(X \times I ; Y \times I,(X \backslash Y) \times I)
$$

where

$$
\begin{gathered}
\partial W=M_{0} \cup M_{1}, \quad \partial V=N_{0} \cup N_{1}, \\
\partial(W \backslash V)=\left(M_{0} \backslash N_{0}\right) \cup\left(M_{1} \backslash N_{1}\right) \cup F^{-1}(\partial Y \times I), \\
F_{M_{i}}=f_{i}, \quad G_{N_{i}}=g_{i}, \quad H_{M_{i} \backslash N_{i}}=h_{i} .
\end{gathered}
$$

Denote by $\mathcal{S}^{T O P}(X, Y)$ the set of concordance classes of $s$-triangulations of the manifold pair $(X, Y, \xi)$.

A simple homotopy equivalence $f: M \rightarrow X$ splits along a submanifold $Y$ if it is homotopy equivalent to an $s$-triangulation of the manifold pair $(X, Y)$. By the definition, every $s$-triangulation $(f ; g, h)$ of the manifold pair $(X, Y)$ defines $s$-triangulations $f$, $g$, and $h$ of the manifolds $X, Y$, and the manifold with boundary $(X \backslash Y, \partial(X \backslash Y))$, respectively.

Thus we obtain natural maps $[11, \S 7.2]$

$$
\begin{aligned}
& \mathcal{S}^{T O P}(X, Y) \rightarrow \mathcal{S}^{T O P}(X), \mathcal{S}^{T O P}(X, Y) \rightarrow \mathcal{S}^{T O P}(Y), \text { and } \\
& \mathcal{S}^{T O P}(X, Y) \rightarrow \mathcal{S}^{T O P}(X \backslash Y, \partial(X \backslash Y)) .
\end{aligned}
$$

Let

$$
F=\left(\begin{array}{ll}
\pi_{1}(S(\xi)) & \rightarrow \pi_{1}(X \backslash Y) \\
\downarrow & \downarrow \\
\pi_{1}(E(\xi)) \rightarrow & \pi_{1}(X)
\end{array}\right)
$$

be a push-out square of fundamental groups with orientations for a manifold pair $(X, Y)$ where $S(\xi)$ is a boundary of a tubular neighborhood $E(\xi)$ of $Y$ in $X$.

Denote by $L S_{n-q}(F), n-q=0,1,2,3 \bmod 4$ the group of obstructions to splitting a simple homotopy equivalence $f: M \rightarrow X$ along the submanifold $Y$ (see $[2,3,6,10,11])$, and let

$$
\Theta^{T O P}: \mathcal{S}^{T O P}(X) \rightarrow L S_{n-q}(F)
$$

be the splitting obstruction map.

Denote also by $L P_{n-q}(F), n=0,1,2,3 \bmod 4$ the group of obstructions to performing surgeries on the closed manifold pair $(X, Y)$ (see $[2,3,6,11,12])$, and let

$$
\mathcal{T}^{T O P}(X) \rightarrow L P_{n-q}(F)
$$

be the corresponding surgery obstruction map. 
The structure set $\mathcal{S}(X, Y)$ and the obstruction groups $L S_{*}(F), L P_{*}(F)$, are realized by the spectra $\mathbb{S}(X, Y), \mathbb{L} S(F)$, and $\mathbb{L} P(F)$ (see $[2,3,6,10,11]$ ) whose homotopy groups are respectively

$$
\begin{aligned}
& \pi_{m}(\mathbb{S}(X, Y))=\mathcal{S}_{m}(X, Y), \pi_{m}(\mathbb{L} S(F))=L S_{m}(F), \pi_{m}(\mathbb{L} P(F))=L P_{m}(F), \\
& \text { and } \pi_{n+1}(\mathbb{S}(X, Y))=\mathcal{S}_{n+1}(X, Y)=\mathcal{S}^{T O P}(X, Y) .
\end{aligned}
$$

The spectra $\mathbb{L} S(F)$ and $\mathbb{L} P(F)$ fit into the homotopy commutative diagram

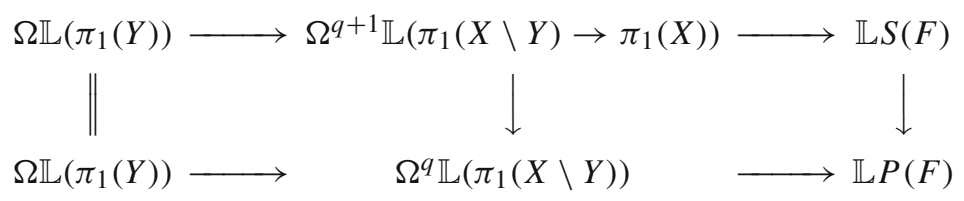

in which the right square is a pullback, the left hand horizontal maps are suspensions of the transfer maps, and the homotopy fiber of the two vertical maps furthest to the right is $\Omega^{q+1} \mathbb{L}\left(\pi_{1}(X)\right)$ (see $[2,3,6,11]$ ).

The homotopy long exact sequences of the maps in (2.13) form a braid of exact sequences

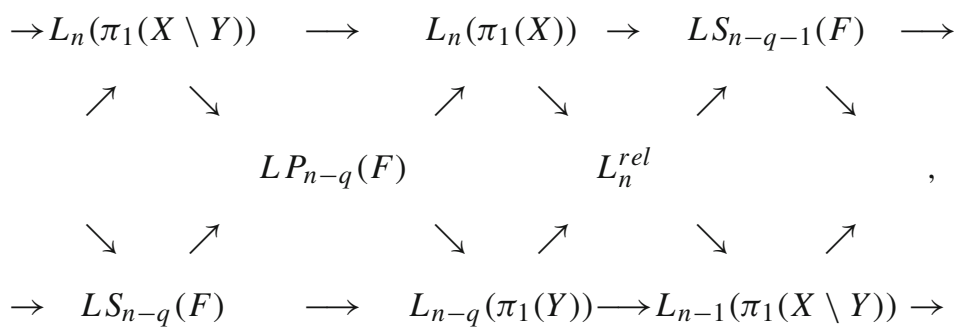

as in [12, page 264], where $L_{n}^{r e l}$ are the relative groups $L_{n}\left(\pi_{1}(X \backslash Y) \rightarrow \pi_{1}(X)\right)$.

The maps in (2.11) and (2.12) are realized on the spectra level by maps fitting into the homotopy commutative diagram of spectra (see [3,6], and [11, §7.2])

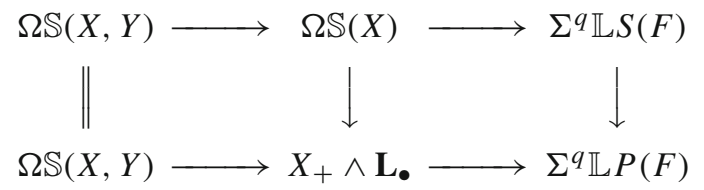

in which the right square is a pullback. The homotopy cofiber of the two vertical maps furthest to the right is $\mathbb{L}\left(\pi_{1}(X)\right)$. 
The homotopy long exact sequences of the maps in the pullback square in (2.15) form a braid of exact sequences [11, Proposition 7.2.6]

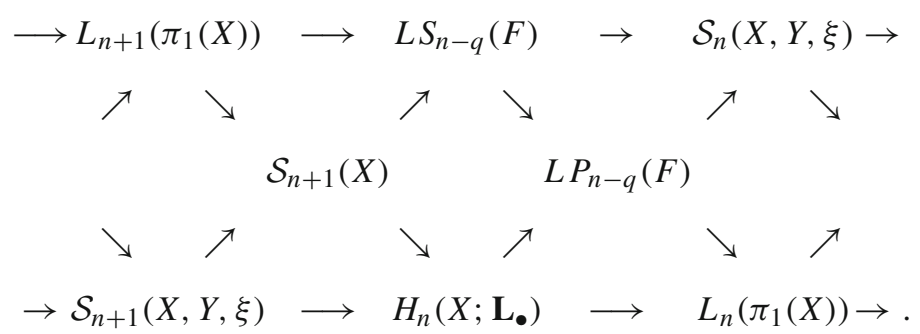

The spectrum $\mathbb{L} P(F)$ fits also into a homotopy commutative diagram (see $[2,3,6]$ )

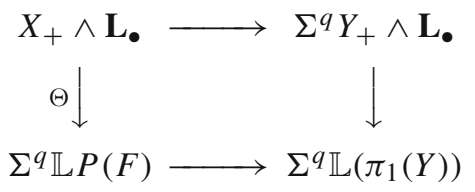

which realizes the diagram in [11, Proposition 7.2.6, iii)]. The other relations between various structure sets and obstruction groups for a closed manifold pair $(X, Y)$ are expressed by many braids of exact sequences, all of which are realized on the spectra level (see $[2,3,6,11])$.

\section{Surgery on two manifolds with a transversal intersection}

In this section we construct the algebraic surgery theory of a stratified space [13, sections 5, 7]

$$
\mathcal{X}=\left(X_{1}, X_{2} ; X_{1} \cap X_{2}=Y\right),
$$

where $X_{1}$ and $X_{2}$ are $n$-dimensional manifolds with a transversal intersection $Y$ of codimension $q$ and $\operatorname{dim} Y=n-q \geq 5$. We shall consider only the case when $X=X_{1} \cup_{Y} X_{2}, X_{1}, X_{2}$, and $Y$ are connected manifolds, but all results will be true in the general case. Let $X=X_{1} \cup_{Y} X_{2}$.

Let $\mathcal{N}=\left(N_{1}, N_{2} ; N_{1} \cap N_{2}=K\right)$ be a stratified space consisting of two transversal manifolds $N_{1}$ and $N_{2}$ with intersection $K$ such that $\operatorname{dim} N_{1}=\operatorname{dim} N_{2}=n, \operatorname{dim} K=$ $n-q$, and $N=N_{1} \cup N_{2}$. Let $\xi_{1}$ be a normal bundle of $Y$ in $X_{1}$, and let $\xi_{2}$ be a normal bundle of $Y$ in $X_{2}$. We have associated to these bundles spherical fibrations [10]

$$
\begin{aligned}
& \left(D^{q}, S^{q-1}\right) \rightarrow\left(E\left(\xi_{1}\right), S\left(\xi_{1}\right)\right) \rightarrow Y, \\
& \left(D^{q}, S^{q-1}\right) \rightarrow\left(E\left(\xi_{2}\right), S\left(\xi_{2}\right)\right) \rightarrow Y .
\end{aligned}
$$


Let $f: K \rightarrow Y$ be a $t$-triangulation of the manifold $Y$. The transfer maps (see [11, $\S 7]$ and $[13, \S 7.2])$

$$
\tau_{1}: \mathcal{T}(Y) \rightarrow \mathcal{T}\left(E\left(\xi_{1}\right), S\left(\xi_{1}\right)\right)
$$

and

$$
\tau_{2}: \mathcal{T}(Y) \rightarrow \mathcal{T}\left(E\left(\xi_{2}\right), S\left(\xi_{2}\right)\right)
$$

define $t$-triangulations

$$
\begin{aligned}
& \left(\tau_{1}(f), \partial \tau_{1}(f)\right):\left(E_{1}, \partial E_{1}\right) \rightarrow\left(E\left(\xi_{1}\right), S\left(\xi_{1}\right)\right), \\
& \left(\tau_{2}(f), \partial \tau_{2}(f)\right):\left(E_{2}, \partial E_{2}\right) \rightarrow\left(E\left(\xi_{2}\right), S\left(\xi_{2}\right)\right)
\end{aligned}
$$

such that the restrictions

$$
\begin{aligned}
& \partial \tau_{1}(f): \partial E_{1} \rightarrow S\left(\xi_{1}\right) \\
& \partial \tau_{2}(f): \partial E_{2} \rightarrow S\left(\xi_{2}\right)
\end{aligned}
$$

are $t$-triangulations of closed manifolds $S\left(\xi_{1}\right)$ and $S\left(\xi_{2}\right)$, respectively.

Definition 3.1 Let

$$
\mathcal{F}=\left(f_{1}, f_{2}, f\right): \mathcal{N} \rightarrow \mathcal{X}
$$

denote a map $N \rightarrow X$ defined by $t$-triangulations

$$
\begin{gathered}
f_{1}: N_{1} \rightarrow X_{1}, \\
f_{2}: N_{2} \rightarrow X_{2}, \\
f: K \rightarrow Y
\end{gathered}
$$

such that $f_{i}(i=1,2)$ is transversal to $Y$ with $f_{i}^{-1}(Y)=K,\left(Y_{i}, N\right)$ is a closed manifold pair with normal block bundle $v_{i}$, and $f=\left.f_{1}\right|_{K}=\left.f_{2}\right|_{K}$. Such a map $\mathcal{F}$ is called $t$-triangulation of the stratified space $\mathcal{X}$, if the constituent maps

$$
\left(f_{i}, f\right):\left(N_{i}, K, v_{i}\right) \rightarrow\left(X_{i}, Y, \xi_{i}\right), i=1,2
$$

are $t$-triangulations of the manifold pairs $\left(X_{i}, Y, \xi_{i}\right)$.

Consider two $t$-triangulations $(\mathcal{F}, \mathcal{N})$ and $(\mathcal{G}, \mathcal{M})$ of a stratified space $\mathcal{X}$ in (3.1), where $\mathcal{N}=\left(N_{1}, N_{2} ; K\right), \mathcal{F}=\left(f_{1}, f_{2} ; f\right), \mathcal{M}=\left(M_{1}, M_{2} ; L\right)$, and $\mathcal{G}=\left(g_{1}, g_{2} ; g\right)$.

Definition 3.2 Two $t$-triangulations $(\mathcal{F}, \mathcal{N})$ and $(\mathcal{G}, \mathcal{M})$ are concordant if the following conditions are satisfied: 
(i) There exists a stratified space $\mathcal{V}=\left(V_{1}, V_{2} ; S=V_{1} \cap V_{2}\right)$ with boundary $\partial \mathcal{V}=\mathcal{N} \cup \mathcal{M}$ such that

$$
\partial V_{1}=N_{1} \dot{\cup} M_{1}, \partial V_{2}=N_{2} \dot{\cup} M_{2}, \partial S=K \dot{U} L
$$

(ii) There exists a stratified map

$$
\Psi=\left(F_{1}, F_{2} ; F\right): \mathcal{V} \rightarrow \mathcal{X} \times I, I=[0,1]
$$

defined by $t$-triangulations

$$
\begin{aligned}
& F_{1}: V_{1} \rightarrow X_{1} \times I, \\
& F_{2}: V_{2} \rightarrow X_{2} \times I, \\
& F=\left.F_{1}\right|_{S}=\left.F_{2}\right|_{S}:: S \rightarrow Y \times I
\end{aligned}
$$

such that $F_{i}$ is transversal to $Y \times I$ and $S=F_{i}^{-1}(Y \times I)(i=1,2)$,

(iii) For $i=1,2$, the pairs $\left(F_{i}, F\right)$ define concordances of the $t$-triangulations $\left(f_{i}, f\right)$ and $\left(g_{i}, g\right)$ of the closed manifold pair $\left(X_{i}, Y\right)$.

Definitions 3.1 and 3.2 are a special case of the notion of $t$-triangulation of a stratified space considered in [11]. The set of concordance classes of $t$-triangulations of $\mathcal{X}$ is denoted by $\mathcal{T}^{T O P}(\mathcal{X})$ and, by Definitions 3.1 and 3.2 , fits into commutative diagram of structure sets

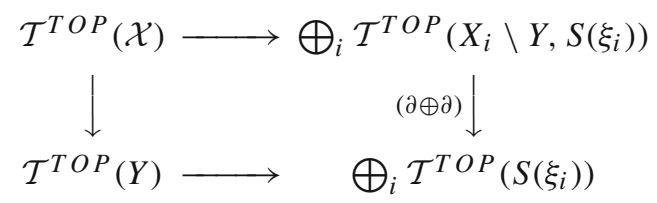

in which the bottom map is the composition

$$
\mathcal{T}^{T O P}(Y) \stackrel{\left(\tau_{1} \top \tau_{2}\right)}{\longrightarrow} \bigoplus_{i=1,2} \mathcal{T}^{T O P}\left(E\left(\xi_{i}\right), S\left(\xi_{i}\right)\right) \stackrel{(\partial \oplus \partial)}{\longrightarrow} \bigoplus_{i=1,2} \mathcal{T}^{T O P}\left(S\left(\xi_{i}\right)\right)
$$

of the transfer maps and the restriction maps and the other maps in the diagram are restrictions of $t$-triangulations.

Define the spectrum $\mathbb{T}(S(\xi))$ by the pullback square of spectra

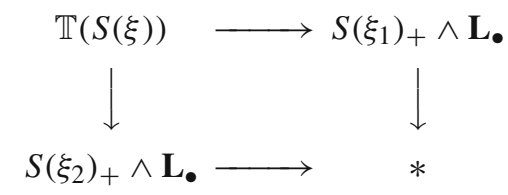

where

$$
\pi_{n-1}\left(S\left(\xi_{1}\right)_{+} \wedge \mathbf{L}_{\bullet}\right)=\mathcal{T}^{T O P}\left(S\left(\xi_{1}\right)\right), \quad \pi_{n-1}\left(S\left(\xi_{2}\right)_{+} \wedge \mathbf{L}_{\bullet}\right)=\mathcal{T}^{T O P}\left(S\left(\xi_{2}\right)\right) .
$$


The spectrum $\mathbb{T}(S(\xi))$ realizes the structure set $\mathcal{T}^{T O P}\left(S\left(\xi_{1}\right)\right) \oplus \mathcal{T}^{T O P}\left(S\left(\xi_{2}\right)\right)$ and has homotopy groups

$$
\pi_{m}(\mathbb{T}(S(\xi)))=H_{m}\left(S\left(\xi_{1}\right) ; L_{\bullet}\right) \oplus H_{m}\left(S\left(\xi_{2}\right) ; L_{\bullet}\right)
$$

such that

$$
\pi_{n-1}(\mathbb{T}(S(\xi)))=\mathcal{T}^{T O P}\left(S\left(\xi_{1}\right)\right) \oplus \mathcal{T}^{T O P}\left(S\left(\xi_{2}\right)\right) .
$$

Define the spectrum $\mathbb{T}(X \backslash Y, S(\xi))$ by the pullback square of spectra

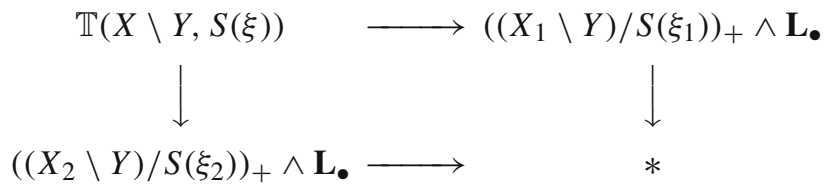

where

$$
\left.\pi_{n}\left(\left(X_{1} \backslash Y\right) / S\left(\xi_{1}\right)\right)_{+} \wedge \mathbf{L}_{\bullet}\right)=\mathcal{T}^{T O P}\left(X_{1} \backslash Y, S\left(\xi_{1}\right)\right)
$$

and

$$
\left.\pi_{n}\left(\left(X_{2} \backslash Y\right) / S\left(\xi_{2}\right)\right)_{+} \wedge \mathbf{L}_{\bullet}\right)=\mathcal{T}^{T O P}\left(X_{2} \backslash Y, S\left(\xi_{2}\right)\right)
$$

The spectrum $\mathbb{T}(X \backslash Y, S(\xi))$ realizes the structure set

$$
\mathcal{T}^{T O P}\left(X_{1} \backslash Y, S\left(\xi_{1}\right)\right) \oplus \mathcal{T}^{T O P}\left(X_{2} \backslash Y, S\left(\xi_{2}\right)\right)
$$

and has homotopy groups

$$
\pi_{m}(\mathbb{T}(X \backslash Y, S(\xi)))=H_{m}\left(X_{1} \backslash Y, S\left(\xi_{1}\right) ; \mathbf{L}_{\bullet}\right) \oplus H_{m}\left(X_{2} \backslash Y, S\left(\xi_{2}\right) ; \mathbf{L}_{\bullet}\right) .
$$

The structure set $\mathcal{T}^{\text {TOP }}(\mathcal{X})$ is realized on the spectra level by a spectrum $\mathbb{T}(\mathcal{X})$ (see $[11, \S 7],[12, \S 17 \mathrm{~A}]$ and $[13, \S 3, \S 7])$ with $\pi_{n}(\mathbb{T}(\mathcal{X}))=\mathcal{T}_{n}(\mathcal{X})=\mathcal{T}^{T O P}(\mathcal{X})$ and fits into the pullback square of spectra

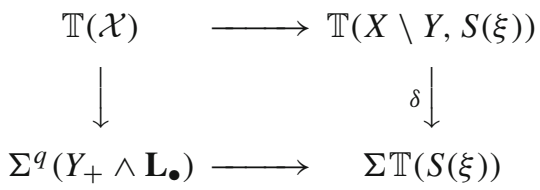

which realizes the diagram of structure sets (3.2). 
It follows immediately from Definitions 3.1 and 3.2 that we have a commutative diagram of structure sets

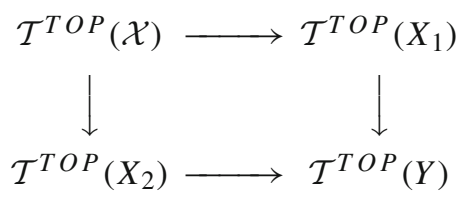

in which all maps are restrictions of $t$-triangulations.

Theorem 3.1 The diagram (3.6) is realized by a pullback square of spectra

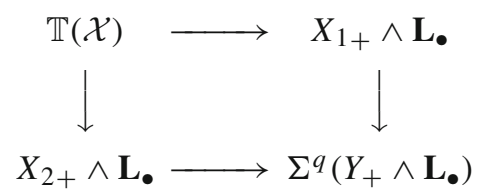

where the bottom horizontal map and the right vertical map are defined as in (2.7).

Proof Denote by $\mathbb{T}(X \backslash Y)$ a spectrum which realizes the structure set $\mathcal{T}^{T O P}\left(X_{1} \backslash\right.$ $Y) \oplus \mathcal{T}^{T O P}\left(X_{2} \backslash Y\right)$. This spectrum fits into the pullback square

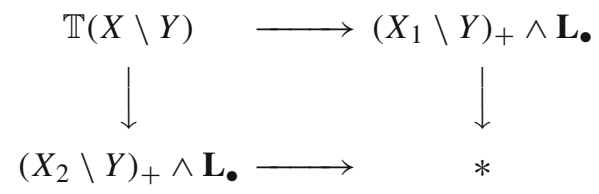

On spectra level, restricting a $t$-triangulation to the boundary provides a natural map $\Delta$ of square (3.4) to the suspended square (3.3) whose cofiber coincides with the suspended square (3.8). The map $\Delta$ in the left upper corner coincides with the map $\delta$ in (3.5). Now from pullback square (3.5) and the cofibrations (2.7) for manifolds pairs $\left(X_{i}, Y\right)(i=1,2)$, we obtain a map of the spectrum $\Sigma^{q}\left(Y_{+} \wedge \mathbf{L}_{\bullet}\right)$ into each of the spectra in the suspended square (3.8) such that the diagram which has the form of a pyramid with top $\Sigma^{q}\left(Y_{+} \wedge \mathbf{L}_{\bullet}\right)$ is homotopy commutative. Now the fibers of the maps from the top of the pyramid to the spectra in the bottom of the pyramid form a pullback square of spectra (3.7). This proves the theorem.

Corollary 3.1 The homotopy long exact sequences of the maps in the pullback square (3.7) form a braid of exact sequences

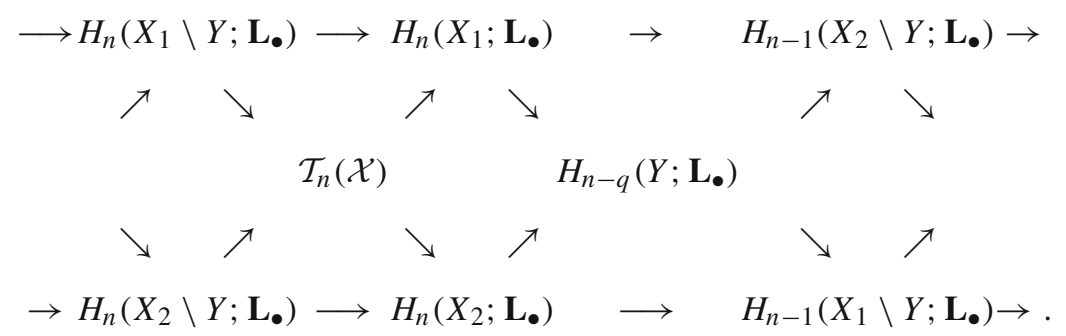


Corollary 3.2 Let $i=1, j=2$ or $i=2, j=1$. The diagram of spectra

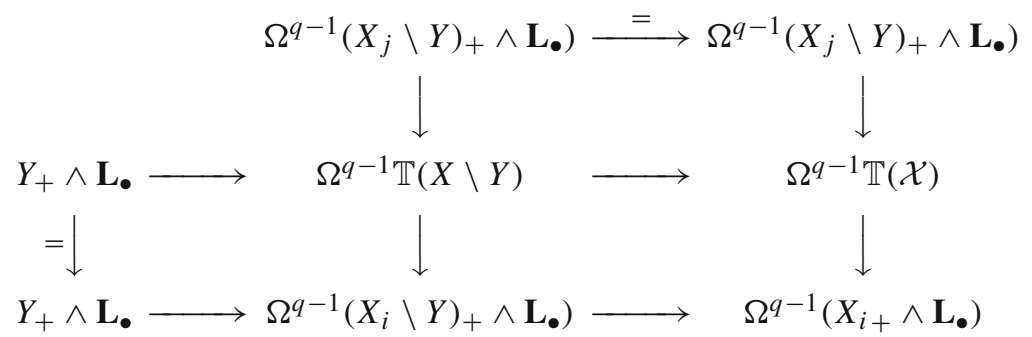

is homotopy commutative and the two bottom rows and two right columns are cofibration sequences. The homotopy long exact sequences of the maps in (3.9) form a braid of exact sequences

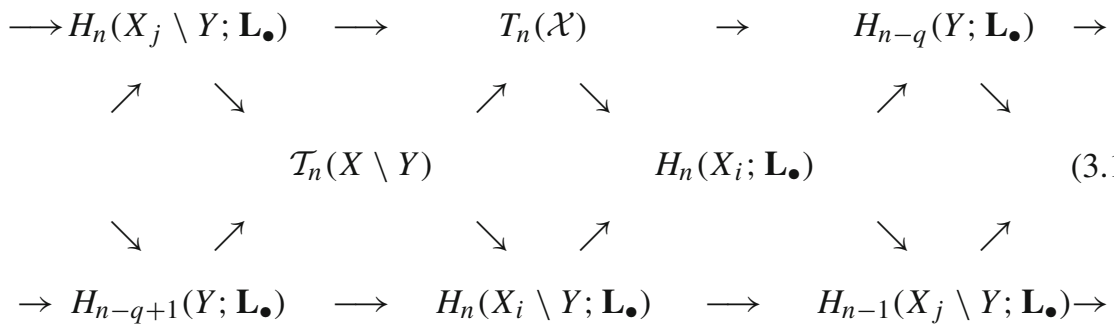

where

$$
\mathcal{T}_{m}(X \backslash Y)=\pi_{m}(\mathbb{T}(X \backslash Y))=H_{m}\left(X_{1} \backslash Y ; \mathbf{L}_{\bullet}\right) \oplus H_{m}\left(X_{2} \backslash Y ; \mathbf{L}_{\bullet}\right) .
$$

Proof Consider the commutative diagram which forms of pyramid in the proof of Theorem 3.1. The left bottom square of (3.9) is the $q$-desuspended part of this diagram for $i=2, j=1$. Hence the right bottom square in (3.9) is a pullback and the result follows. Note that the two right bottom vertical maps in (3.9) geometrically correspond to restricting a $t$-triangulation of the manifold with boundary $X \backslash Y$ to a $t$-triangulation of the manifold with boundary $X_{i} \backslash Y$ and to restricting a $t$-triangulation of $\mathcal{X}$ to a $t$-triangulation of the manifold $X_{i}$, respectively.

It is worth noting the braids of exact sequences obtained in Corollaries 3.1 and 3.2 together with the Atiyah-Hirzebruch spectral sequence for $H_{n}\left(? ; \mathbf{L}_{\bullet}\right)$ [1] provide a tool for computing the structure sets $\mathcal{T}_{i}(\mathcal{X})$.

Using (3.1), we define now a spectrum $\mathbb{L}(\mathcal{X})$ for surgery obstruction groups of a stratified space $\mathcal{X}$ (see $[13, \S 6])$.

For $i=1,2$, let $\partial \tau_{i}$ denote the composition of the maps

$$
L_{m}\left(\pi_{1}(Y)\right) \stackrel{\tau_{i}}{\longrightarrow} L_{m+q}\left(\pi_{1}\left(S\left(\xi_{i}\right)\right) \rightarrow \pi_{1}\left(E\left(\xi_{i}\right)\right)\right) \stackrel{\partial}{\longrightarrow} L_{m+q-1}\left(\pi_{1}\left(S\left(\xi_{i}\right)\right)\right)
$$


where the first map is the transfer map and the second is the boundary map in the relative long exact sequence of $L$-groups for the map $\pi_{1}\left(S\left(\xi_{i}\right)\right) \rightarrow \pi_{1}\left(E\left(\xi_{i}\right)\right.$ ) (see $[2,3,10,11])$. For future use below, we record the diagram of groups

$$
\begin{aligned}
& L_{m}\left(\pi_{1}(Y)\right) \stackrel{\left(\partial \tau_{1} \partial \tau_{2}\right)}{\longrightarrow} \quad L_{m+q-1}\left(\pi _ { 1 } ( S ( \xi _ { 1 } ) ) \oplus L _ { m + q - 1 } \left(\pi_{1}\left(S\left(\xi_{2}\right)\right)\right.\right. \\
& \left\lfloor i_{1 *} \oplus i_{2 *}\right. \\
& L_{m+q-1}\left(\pi_{1}\left(X_{1} \backslash Y\right)\right) \oplus L_{m+q-1}\left(\pi_{1}\left(X_{2} \backslash Y\right)\right)
\end{aligned}
$$

where the first map is given by (3.11) and the second is induced by the inclusions $i_{1}: S\left(\xi_{1}\right) \rightarrow\left(X_{1} \backslash Y\right)$ and $i_{2}: S\left(\xi_{2}\right) \rightarrow\left(X_{2} \backslash Y\right)$. Note that all maps in (3.11) and (3.12) are realized on the spectra level.

Define the spectrum $\mathbb{L}(X \backslash Y)$ with homotopy groups

$$
\pi_{m}(\mathbb{L}(X \backslash Y))=L_{m}\left(\pi_{1}\left(X_{1} \backslash Y\right)\right) \oplus L_{m}\left(\pi_{1}\left(X_{2} \backslash Y\right)\right)
$$

by the pullback square

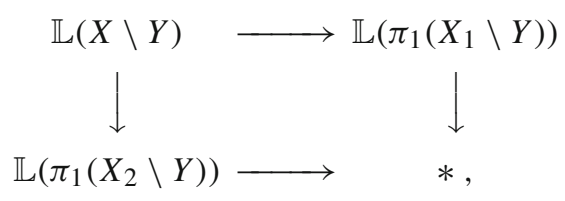

and define the spectrum $\mathbb{L}(S(\xi)))$ with homotopy groups

$$
\pi_{m}(\mathbb{L}(S(\xi)))=L_{m}\left(\pi_{1}\left(S\left(\xi_{1}\right)\right)\right) \oplus L_{m}\left(\pi_{1}\left(S\left(\xi_{2}\right)\right)\right)
$$

by the pullback square

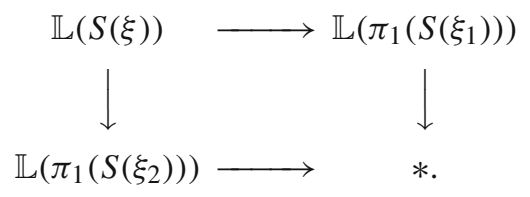

The diagram (3.12) can be written on the spectra level in the form

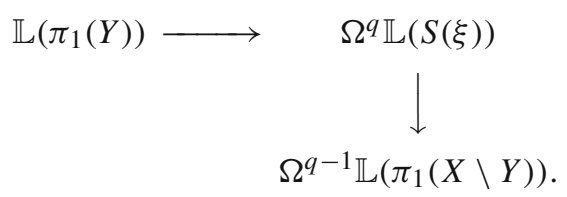

We denote the composition of the maps in (3.13) by d. Let $\mathbb{L}(\mathcal{X})$ denote the homotopy fiber of the map $\mathbf{d}$. It fits into a cofibration sequence (see $[13, \S 6]$ )

$$
\mathbb{L}(\mathcal{X}) \longrightarrow \mathbb{L}\left(\pi_{1}(Y)\right) \stackrel{\mathbf{d}}{\longrightarrow} \Omega^{q-1} \mathbb{L}(X \backslash Y) .
$$


Let $F_{i}(i=1,2)$ denote the square of fundamental groups in the splitting problem for the closed manifold pair $Y \subset X_{i}$.

Theorem 3.2 The spectrum $\mathbb{L}(\mathcal{X})$ fits into a pullback square

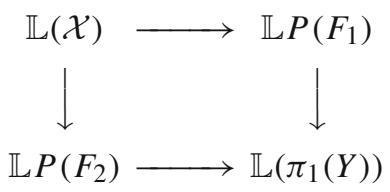

of spectra and the homotopy long exact sequences of the maps in (3.15) form a braid of exact sequences

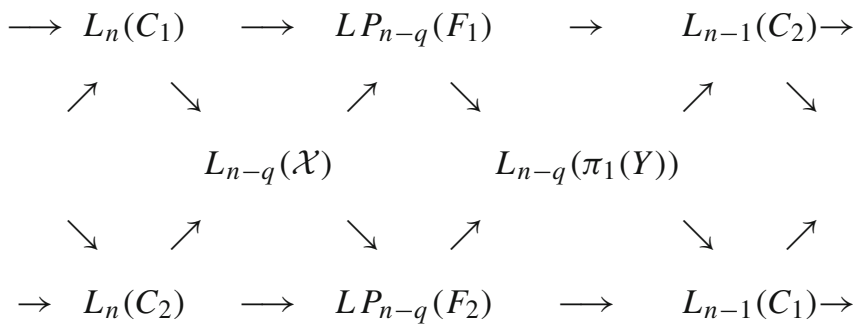

where $C_{i}=\pi_{1}\left(X_{i} \backslash Y\right)(i=1,2)$.

Proof The maps in diagram (3.13) define a map of the spectrum $\mathbb{L}\left(\pi_{1}(Y)\right)$ into the pullback square

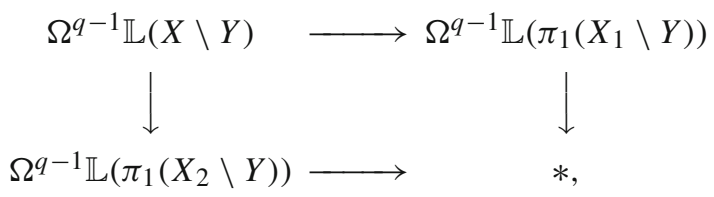

and we obtain a homotopy commutative diagram in the form of a pyramid whose base is the diagram in (3.17). By (2.13) and (3.14) the fibers of the maps from $\mathbb{L}\left(\pi_{1}(Y)\right)$ to the spectra in the base of the pyramid form a pullback square of spectra (3.15). The homotopy long exact sequences of the maps in (3.15) form the braid exact sequences (3.16).

Corollary 3.3 Let $i=1, j=2$ or $i=2, j=1$. The diagram of spectra

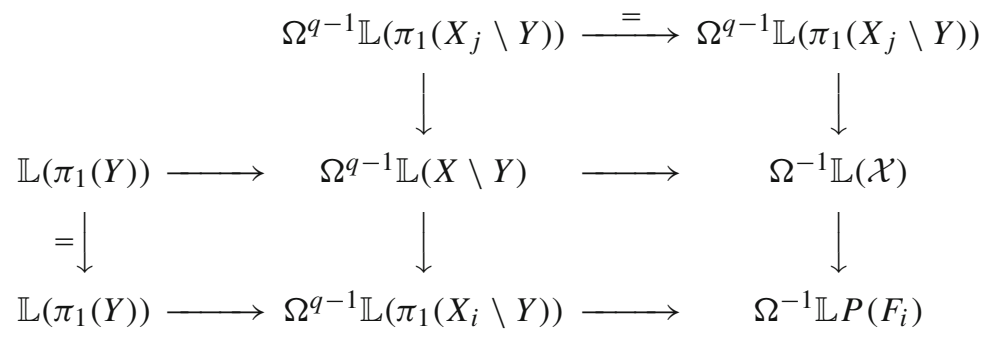


is homotopy commutative and the two bottom rows and two right columns are cofibration sequences. The homotopy long exact sequences of the maps in (3.18) form a braid of exact sequences

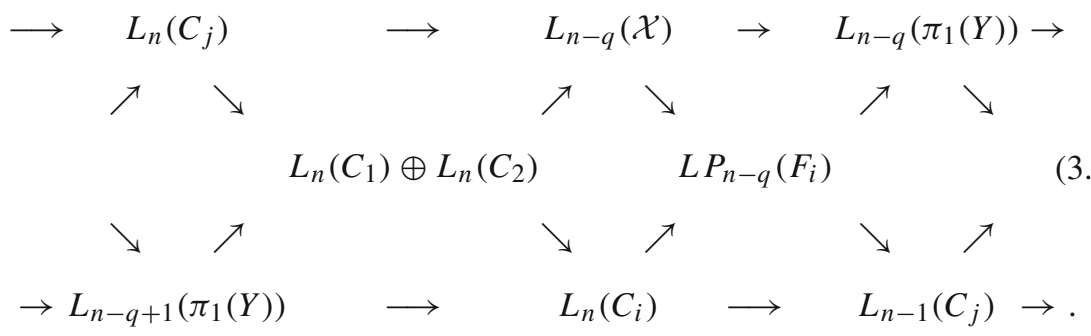

Proof Similar to that of Corollary 3.2.

Theorem 3.3 Let $i=1, j=2$ or $i=2, j=1$. The diagram of spectra

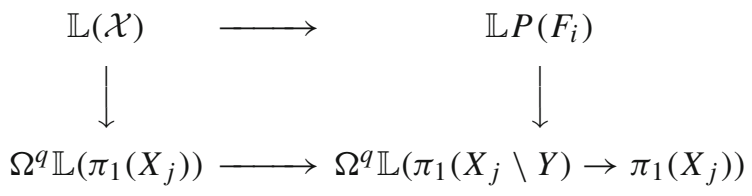

is a pullback diagram. The homotopy long exact sequences of the horizontal maps in (3.20) form a commutative diagram

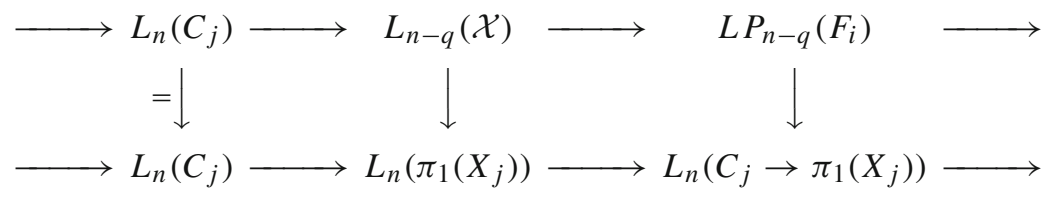

where $C_{j}=\pi_{1}\left(X_{j} \backslash Y\right)$.

Proof Consider the homotopy commutative diagram of spectra

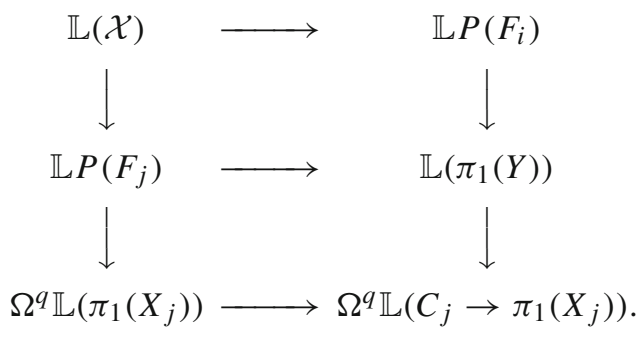

The upper square is (3.15) and the bottom square is the central square in(2.14) on the spectra level. The squares in (3.22) are pullback squares and the fibers of all horizontal maps are naturally homotopy equivalent to $\Omega^{q} \mathbb{L}\left(C_{j}\right)$. 
Definition $3.3([13, \S 7])$ A $t$-triangulation

$$
\mathcal{F}=\left(f_{1}, f_{2}, f\right): \mathcal{N} \rightarrow \mathcal{X}
$$

of the stratified space $\mathcal{X}$ (see 3.1) is called an $s$-triangulation, if $\mathcal{F}$ is given by a simple homotopy equivalence $N \rightarrow X$ and the constituent maps

$$
\left(f_{i}, f\right):\left(N_{i}, K, v_{i}\right) \rightarrow\left(X_{i}, Y, \xi_{i}\right), i=1,2
$$

are $s$-triangulations of the closed manifold pairs $\left(X_{i}, Y, \xi_{i}\right)$.

The definition of concordance of $s$-triangulations of a stratified space is similar to concordance of $t$-triangulations of stratified space in Definition 3.2.

Definition 3.4 Two $s$-triangulations $(\mathcal{F}, \mathcal{N})$ and $(\mathcal{G}, \mathcal{M})$ of $\mathcal{X}$ are concordant, if the following conditions are satisfied:

(i) There exists a concordance

$$
\Psi=\left(F_{1}, F_{2} ; F\right): \mathcal{V}=\left(V_{1}, V_{2} ; S=V_{1} \cap V_{2}\right) \rightarrow \mathcal{X} \times I, I=[0,1]
$$

of the $t$-triangulations $(\mathcal{F}, \mathcal{N})$ and $(\mathcal{G}, \mathcal{M})$ such that the map

$$
\psi=\Psi_{V_{1} \cup_{S} V_{2}}: V_{1} \cup_{S} V_{2} \rightarrow X \times I
$$

is a simple homotopy equivalence.

(ii) For $i=1,2$, the pair $\left(F_{i}, F\right)$ is a concordance of the $s$-triangulations $\left(f_{i}, f\right)$ and $\left(g_{i}, g\right)$ of the closed manifold pair $\left(X_{i}, Y\right)$.

The set of concordance classes of $s$-triangulations is denoted by $\mathcal{S}^{T O P}(\mathcal{X})$ and is realized by a spectrum $\mathbb{S}(\mathcal{X})$ with homotopy groups $\pi_{m}(\mathbb{S}(\mathcal{X}))=\mathcal{S}_{m}(\mathcal{X})$ such that $\mathcal{S}^{T O P}(\mathcal{X})=\mathcal{S}_{n+1}(\mathcal{X}), n=\operatorname{dim} X_{i}$ [11]. Letting $\mathbb{T}(\mathcal{X})$ be as in (3.5), we obtain a cofibration sequence of spectra [11]

$$
\mathbb{T}(\mathcal{X}) \rightarrow \Sigma^{q} \mathbb{L}(\mathcal{X}) \rightarrow \mathbb{S}(\mathcal{X})
$$

whose homotopy long exact sequence is

$$
\cdots \rightarrow \mathcal{S}_{n+1}(\mathcal{X}) \rightarrow \mathcal{T}_{n}(\mathcal{X}) \rightarrow L_{n-q}(\mathcal{X}) \rightarrow \mathcal{S}_{n}(\mathcal{X}) \rightarrow \ldots
$$

where $n$ is the dimension of the ambient manifolds $X_{i}(i=1,2)$. Recall that $\mathcal{T}_{n}(\mathcal{X})=$ $\mathcal{T}^{T O P}(\mathcal{X})$ and that we are using the convention that the dimension of the surgery obstruction group of a $t$-triangulation of $\mathcal{X}$ is the dimension of the submanifold $Y$. It follows immediately from Definitions 3.3 and 3.4 that the spectrum $\mathbb{S}(\mathcal{X})$ fits into a homotopy commutative diagram of spectra

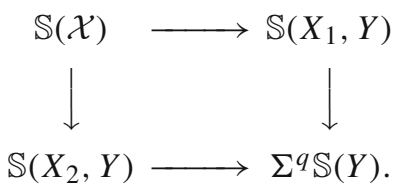


Theorem 3.4 The square in (3.25) is a pullback square. The fiber of the vertical maps is $\mathbb{S}\left(X_{1} \backslash Y\right)$ and the fiber of the horizontal maps is $\mathbb{S}\left(X_{2} \backslash Y\right)$. The homotopy long exact sequences of the maps in (3.25) form a braid of exact sequences

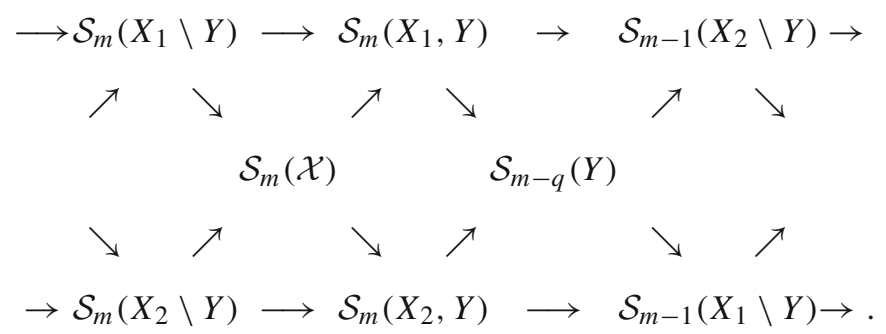

Proof By (2.17), we get a homotopy commutative diagram

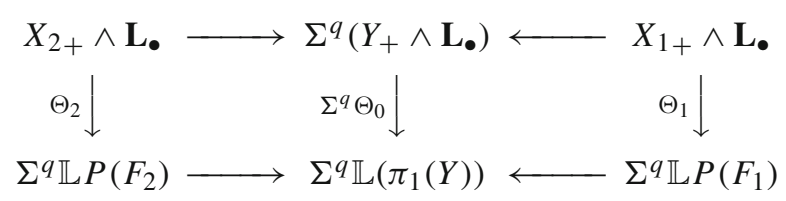

where the squares are defined by the closed manifold pairs $\left(X_{i}, Y\right)$ and $q$ denotes the codimension of $Y$ in $X_{i}$. The vertical maps in (3.27) induce the map

$$
\mathbb{T}(\mathcal{X}) \rightarrow \Sigma^{q} \mathbb{L}(\mathcal{X})
$$

in the cofibration (3.23). Thus we obtain a map $\Lambda$ of the square (3.7) to the $q$-suspended square (3.15). This map is given by a commutative diagram which has the form of a cube. The homotopy cofibers of the maps from the corners of the square (3.7) to the corresponding corners of the pullback square (3.15) form the pullback square (3.25). The theorem follows.

Define the spectrum $\mathbb{S}(X \backslash Y)$ by the pullback square

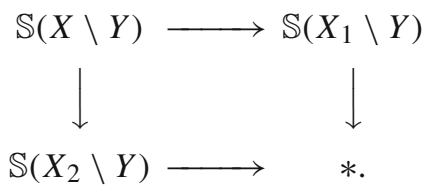

The spectrum $\mathbb{S}(X \backslash Y)$ has the homotopy groups

$$
\mathcal{S}_{m}(X \backslash Y)=\pi_{m}(\mathbb{S}(X \backslash Y))=S_{m}\left(X_{1} \backslash Y\right) \oplus S_{m}\left(X_{2} \backslash Y\right)
$$


Theorem 3.5 The diagram of spectra

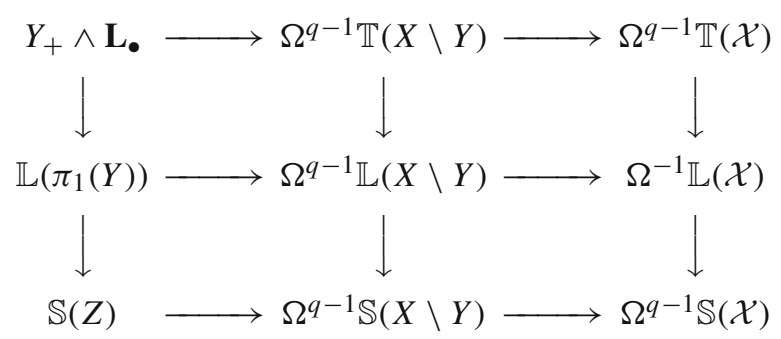

is homotopy commutative and the rows and columns are cofibrations. Let $C_{i}=\pi_{1}\left(X_{i} \backslash\right.$ $Y)$ for $i=1,2$. The homotopy long exact sequences of the maps in (3.28) form a commutative diagram

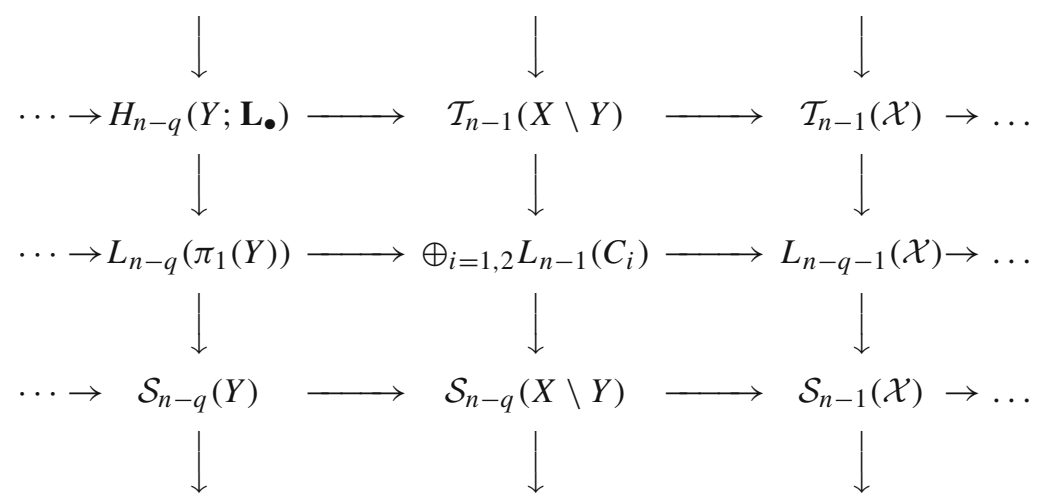

whose rows and columns are exact.

Proof The upper row of diagram (3.28) is the middle row of diagram (3.9), and the middle row of diagram (3.28) is the middle row of diagram (3.18). The upper vertical maps in (3.28) are the surgery obstruction maps on the spectra level. The theorem follows.

Theorem 3.6 Let $C_{i}=\pi_{1}\left(X_{i} \backslash Y\right)$ for $i=1,2$, and $i=1, j=2$ or $i=2, j=1$. The diagram of groups

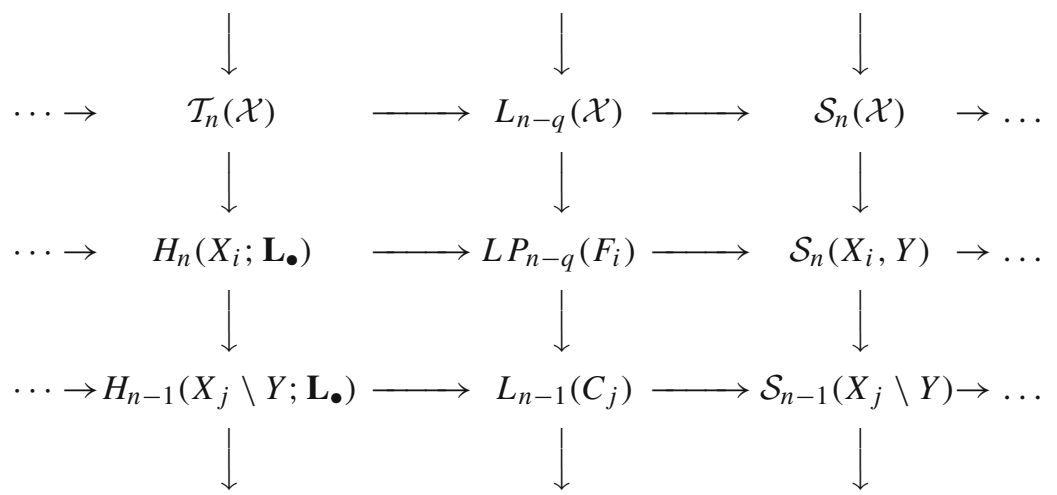


is commutative and its rows and columns are exact.

For $i=1,2$, the is diagram of groups

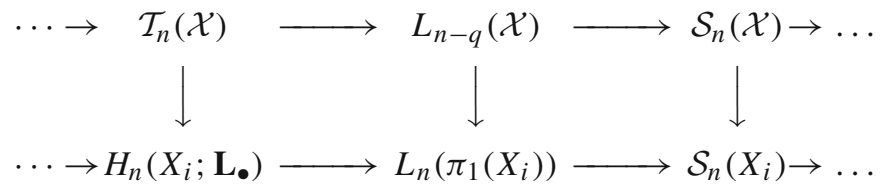

is commutative and its rows are exact. The diagrams (3.30) and (3.31) are realized on the spectra level.

Proof For $i=1,2$, consider the homotopy commutative square of spectra

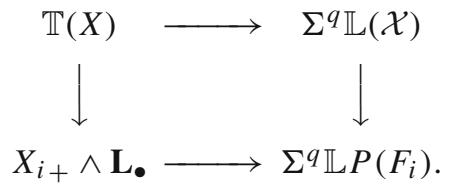

The horizontal maps are the surgery obstruction maps on the spectra level. The vertical maps are described in Theorems 3.1 and 3.2. The square extends to an infinite homotopy commutative bicomplex of spectra which is the realization of the diagram (3.30) on the spectra level. This proves the assertion of the theorem regarding the diagram (3.30). The assertion for diagram (3.31) is proved similarly.

Theorem 3.7 Let $i=1, j=2$ or $i=2, j=1$. The diagram of spectra

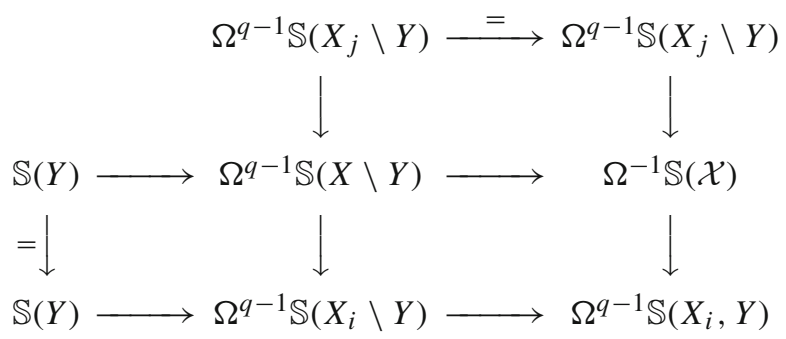

is homotopy commutative and its rows and columns are cofibration sequences. The homotopy long exact sequences of the maps in (3.33) form a braid of exact sequences

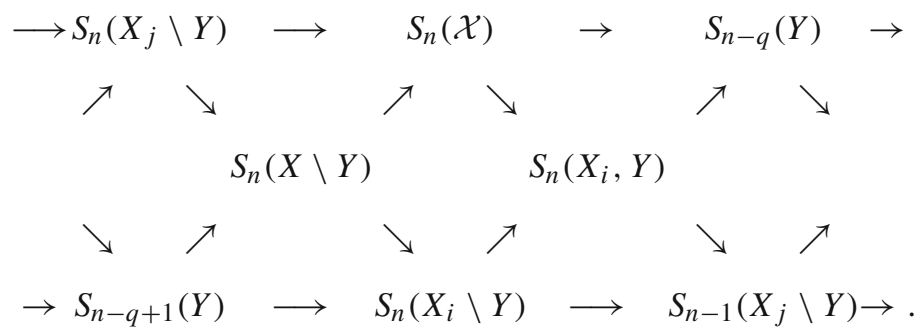


Proof On the spectra level, consider surgery obstruction maps from the spectra in diagram (3.9) to the corresponding spectra in diagram (3.18). The cofibers of these maps form the homotopy commutative diagram (3.33).

\section{An example}

In this section we compute the structure sets of a stratified manifold consisting of two real projective spaces which intersect transversally. Commutative diagrams in Sect. 3 allow us to obtain very explicit results.

Consider a stratified manifold $\mathcal{X}$ as in (3.1) where $X_{i}=\mathbb{R} P^{n}(i=1,2)$ and $Y=X_{1} \cap X_{2}=\mathbb{R} P^{n-1}$. We shall suppose that $n \geq 6$. There are isomorphisms [7, pp. 48, 53] ( see also [12, §14D])

$$
\mathcal{T}^{T O P}\left(\mathbb{R} P^{2 i+5}\right)=\mathcal{T}^{\text {TOP }}\left(\mathbb{R} P^{2 i+4}\right)=\bigoplus_{j=1}^{i+2} \mathbb{Z}_{2}
$$

and

$$
\mathcal{S}^{T O P}\left(\mathbb{R} P^{n}\right)=\bigoplus_{j=1}^{2 k} \mathbb{Z}_{2}, \bigoplus_{j=1}^{2 k} \mathbb{Z}_{2}, \quad \bigoplus_{j=1}^{2 k} \mathbb{Z}_{2} \oplus \mathbb{Z}, \quad \bigoplus_{j=1}^{2 k+1} \mathbb{Z}_{2}
$$

where in (4.2), $k$ is the largest integer such that $4 k+1 \leq n$. The manifold with boundary

$$
X_{i} \backslash Y=\mathbb{R} P^{n} \backslash \mathbb{R} P^{n-1}(i=1,2)
$$

is a disk $D^{n}$, hence

$$
H_{n}\left(X_{i} \backslash Y ; \mathbf{L}_{\bullet}\right)=L_{n}(1)=\mathbb{Z}, 0, \mathbb{Z}_{2}, \quad 0
$$

for $n=0,1,2,3 \bmod 4$, respectively.

Theorem 4.1 Let

$$
\mathcal{X}=\left(\mathbb{R} P^{n}, \mathbb{R} P^{n} ; \mathbb{R} P^{n-1}\right), \quad n \geq 6
$$

Then

$$
\begin{aligned}
& \mathcal{T}^{\text {TOP }}(\mathcal{X}) \cong \mathcal{T}^{\text {TOP }}\left(\mathbb{R} P^{4 k+1}\right)=\bigoplus_{j=1}^{2 k} \mathbb{Z}_{2}, \\
& \mathcal{T}^{\text {TOP }}(\mathcal{X}) \cong \mathcal{T}^{\text {TOP }}\left(\mathbb{R} P^{4 k+2}\right) \oplus \mathbb{Z}_{2}=\bigoplus_{j=1}^{2 k+2} \mathbb{Z}_{2},
\end{aligned}
$$




$$
\begin{aligned}
& \mathcal{T}^{\text {TOP }}(\mathcal{X}) \cong \mathcal{T}^{\text {TOP }}\left(\mathbb{R} P^{4 k+3}\right)=\bigoplus_{j=1}^{2 k+1} \mathbb{Z}_{2} \\
& \mathcal{T}^{\text {TOP }}(\mathcal{X}) \cong \mathcal{T}^{\text {TOP }}\left(\mathbb{R} P^{4 k+4}\right) \oplus \mathbb{Z}_{2}=\bigoplus_{j=1}^{2 k+3} \mathbb{Z}_{2},
\end{aligned}
$$

for $n=4 k+1,4 k+2,4 k+3,4 k+4$, respectively.

Proof In dimensions $4 k+1,4 k+2$, and $4 k+3$, the result follows from Corollary 3.1 and (4.1). In dimension $4 k+4$, it is necessary additionally to use the fact that $\operatorname{Ext}_{\mathbb{Z}}\left(\mathbb{Z}_{2}, \mathbb{Z}\right)=\mathbb{Z} / 2 \mathbb{Z}$.

Theorem 4.2 Let

$$
\mathcal{X}=\left(\mathbb{R} P^{n}, \mathbb{R} P^{n} ; \mathbb{R} P^{n-1}\right), \quad n \geq 6
$$

Then there are isomorphisms

$$
\mathcal{S}^{T O P}(\mathcal{X}) \cong \mathcal{S}^{T O P}\left(\mathbb{R} P^{n}, \mathbb{R} P^{n-1}\right) \cong \mathcal{S}^{T O P}\left(\mathbb{R} P^{n-1}\right)
$$

Proof The braid (3.26) provides the isomorphisms in (4.4), since $\mathcal{S}_{m}\left(p t_{*}\right)=0[10$, page 276].

Acknowledgments Partially supported by the CONACyT Grant 98697. The second author acknowledges the support of a DAAD Fellowship to visit the University of Bielefeld.

\section{References}

1. Adams, J.F.: Stable homotopy and generalised homology. In: Chicago Lectures in Mathematics. The University of Chicago Press, Chicago (1974)

2. Bak, A., Muranov, Y.V.: Splitting along submanifolds and L-spectra. J. Math. Sci. (N.Y) 123(4), 4169-4184 (2004)

3. Bak, A., Muranov, Y.V.: Splitting a simple homotopy equivalence along a submanifold with filtration Matem. Sbornik 199(6), 3-26 (2008). English transl. in Sbornik: Math. 199, 787-809 (2008)

4. Bak, A., Muranov, Y.V.: Normal invariants of manifold pairs and assembly maps Matem. Sbornik 197(6), 3-24 (2006). English transl. in Sbornik: Math. 197, 791-811 (2006)

5. Browder, W., Quinn, F.: A surgery theory for G-manifolds and stratified spaces. In: Manifolds, pp. 27-36. Univ. of Tokyo Press, Tokyo (1975)

6. Cencelj, M., Muranov, Y.V., Repovš, D.: On structure sets of manifold pairs. Homol. Homotopy Appl. 11(2), 195-222 (2009)

7. Lopezde Medrano, S.: Involutions on Manifolds. Springer, Berlin (1971)

8. Milnor, J.: Lectures on the $h$-cobordism theorem. Notes by L. Siebenmann and J. Sondow. Princeton University Press, Princeton (1965)

9. Muranov, Y.V.: Splitting obstruction groups and quadratic extensions of anti-structures Izvestiya: Mathematics 59(6), 1207-1232 (1995). English transl. from Izvestiya RAN: Ser. Mat. 59(6), 107-132

10. Ranicki, A.A.: The total surgery obstruction. In: Lecture Notes in Math., vol. 763, pp. 275-316. Springer, Berlin (1979)

11. Ranicki, A.A.: Exact sequences in the algebraic theory of surgery. In: Math. Notes 26. Princeton Univ. Press, Princeton (1981) 
12. Wall, C.T.C.: Ranicki, A.A. (ed.) Surgery on Compact Manifolds. Academic Press, London (1970); 2nd edn. Amer. Math. Soc., Providence 1999

13. Weinberger, S.: The Topological Classification of Stratified Spaces. The university of Chicago Press, Chicago (1994) 